\title{
EXCITON MAGNETIC POLARON FEATURES IN PHOTOLUMINESCENCE EXCITATION SPECTRA OF CdTe/(CdMn)Te QUANTUM WELLS WITH HIGH Mn CONTENTS*
}

\author{
G. Mackh, W. Ossau, D.R. Yakovlev ${ }^{\dagger}$, G. Landwehr \\ Physikalisches Institut der Universität Würzburg \\ Am Hubland, 97074 Würzburg, Germany \\ T. Wojtowicz, G. Karczewsin and J. Kossut \\ Institute of Physics, Polish Academy of Sciences \\ Al. Lotników 32/46, 02-668 Warszawa, Poland
}

\begin{abstract}
Exciton magnetic polarons are studied in $\mathrm{CdTe} / \mathrm{Cd}_{1-x} \mathrm{Mn}_{x} \mathrm{Te}(0.4 \leq$ $x \leq 0.8)$ quantum wells. The magnetic polaron formation leads to the appearance of an additional line in the photoluminescence excitation spectra, which can be employed to determine the Zeeman splittings more exactly than by using the free exciton peak. We find an overall increase in the polaron energy with increasing $x$ in the whole range of $\mathrm{Mn}$ contents studied.
\end{abstract}

PACS numbers: 78.55.Et, 71.35.+z

In narrow $\mathrm{CdTe} / \mathrm{Cd}_{1-x} \mathrm{Mn}_{x} \mathrm{Te}$ quantum wells (QWs) two-dimensional exciton magnetic polarons (MPs) are formed from excitons confined in nonmagnetic CdTe layers due to the penetration of the exciton wave function into the semimagnetic barrier layers [1]. MP energies and the Zeeman splittings of the free excitons reflect sensitively the magnetic properties of the interfaces $[2,3]$. $\mathrm{CdTe} / \mathrm{Cd}_{1-x} \mathrm{Mn}_{x} \mathrm{Te}$ heterostructures with high $\mathrm{Mn}$ contents are particularly interesting because of the spin-glass phase at low temperatures and the large band gap discontinuity, but the determination of the Zeeman splittings becomes more difficult for high $\mathrm{Mn}$ contents due to the inhomogeneous broadening of the exciton lines. We studied narrow $\mathrm{CdTe} / \mathrm{Cd}_{1-x} \mathrm{Mn}_{x} \mathrm{Te} \mathrm{QWs}$ with high $x(0.4 \leq x \leq 0.8)$. The structures were grown on GaAs substrates by molecular beam epitaxy, which allows one to produce high quality $\mathrm{Cd}_{1-x} \mathrm{Mn}_{x}$ Te with cubic zinc-blende structure for $x$ up to 1 [4].

${ }^{*}$ This work has been partly supported by the DFG through grant SFB410, by the Volkswagen Foundation and in Poland by the State Committee for Scientific Research (Republic of Poland) under grant PBZ-Z011/P4/93/01.

tOn leave from A.F. Ioffe Physico-Technical Institute, St. Petersburg, Russia. 


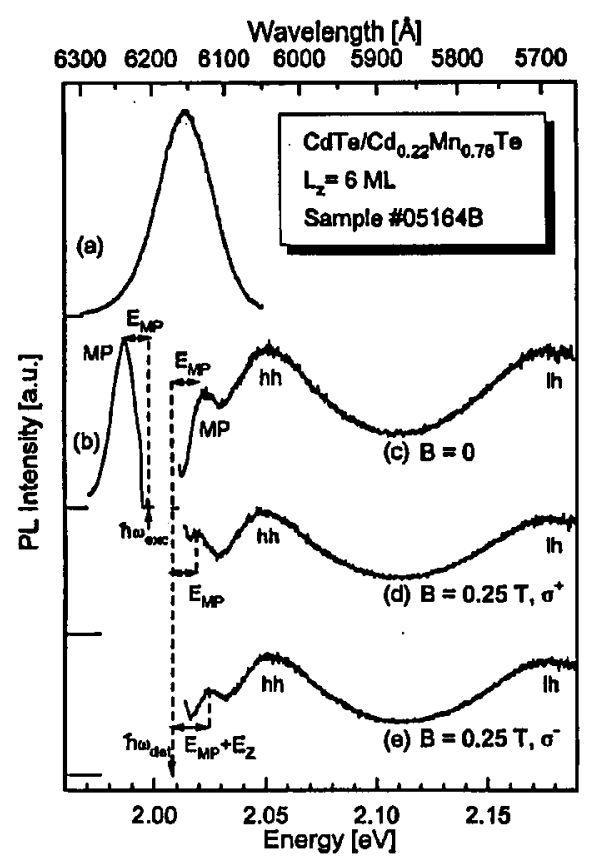

Fig. 1. Photoluminescence spectrum of a 6-ML-wide $(20 \AA) \mathrm{CdTe} / \mathrm{Cd}_{0.22} \mathrm{Mn}_{0.78} \mathrm{Te}$ single quantum well excited at $2.41 \mathrm{eV} ; T=1.6 \mathrm{~K}$. (b) Selective excitation at $\hbar \omega_{\text {exc }}=$ $1.997 \mathrm{eV}$. (c)-(e) Photoluminescence excitation spectra detected at $\hbar \omega_{\text {det }}=2.009 \mathrm{eV}$ at (c) $B=0$, linearly polarized excitation, (d) $B=0.25 \mathrm{~T}$, right-circularly polarized $\left(\sigma^{+}\right)$ excitation, (e) $B=0.25 \mathrm{~T}$, left-circularly polarized $\left(\sigma^{-}\right)$excitation.

The energies of the exciton MPs were determined by the method of selective excitation [1]. Figure 1(a) shows the PL spectrum of a 6 -monolayer (ML)-wide $\mathrm{CdTe} / \mathrm{Cd}_{0.22} \mathrm{Mn}_{0.78} \mathrm{Te} \mathrm{QW}$ excited at $2.41 \mathrm{eV}$ and (b) the spectrum under selective excitation at $1.997 \mathrm{eV}$ with the peak Stokes-shifted by the MP energy $E_{\mathrm{MP}}=10 \mathrm{meV}$. Spectrum (c) is a PL excitation (PLE) spectrum detected at $2.009 \mathrm{eV}$, which clearly shows the heavy-hole- (hh) and light-hole- (lh) exciton peaks. The strong quantum confinement of carriers and the strain lead to the large splitting of the lh- and hh-states of about $125 \mathrm{meV}$. The large Stokes shift between the PL maximum (a) and the hh-exciton-resonance in the PLE spectrum (c) of more than $35 \mathrm{meV}$ results from the exciton localization at well-width fluctuations and, to a minor extent, from the MP formation. The former contribution is particularly important in wells with high barriers. The peak at $2.025 \mathrm{eV}$ in spectrum (c) follows changes of the detection wavelength with a constant energy

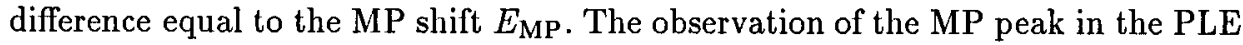
spectrum is due to the fact that the nonmagnetic localization potential caused by well-width fluctuations is large compared to the MP energy, which is typical for QWs with high barriers. Spectra (d) and (e) were detected at the same energy as (c), but are taken at a magnetic field of $B=0.25 \mathrm{~T}$ (Faraday-geometry) under 
right- and left-circularly polarized excitation, respectively. With increasing magnetic field the energy difference between the MP related feature and the detection energy decreases for right-circularly polarized $\left(\sigma^{+}\right)$excitation (d) and increases for left-circularly polarized $\left(\sigma^{-}\right)$excitation (e). $\sigma^{-}$-excitation creates excitons with spin orientation antiparallel to the magnetic field direction. Most of these excitons first relax to the lower $\sigma^{+}$-branch and subsequently form MPs oriented parallel to the magnetic field direction. In this case the energy difference between peak and detection energy is due to both MP formation and Zeeman splitting. Therefore, in these structures the total hh-exciton Zeeman splitting can be determined from the energy difference between the MP features in the PLE spectra taken under $\sigma^{+}$and $\sigma^{-}$excitation. Note that the linewidths of the MP features in spectra (d) and (e) are considerably smaller than the widths of the hh-exciton-lines. Hence, employing the MP features allows one to determine the hh-exciton Zeeman splitting more exactly than via the energy position of the hh-exciton lines. In Fig. 2a we show the total Zeeman splitting determined from the hh-exciton peaks (open circles) and from the MP features in the PLE spectra (closed circles). The accuracy is shown by error bars. Both methods yield nearly equal results. The Zeeman splitting was fitted according to Ref. [2] with the parameters $S_{0}=0.6$ and $T_{\text {eff }}=5 \mathrm{~K}$. We note that these parameters evidence a stronger paramagnetic behavior of the interface spins compared to the values expected for bulk $\mathrm{Cd}_{0.22} \mathrm{Mn}_{0.78} \mathrm{Te}$.
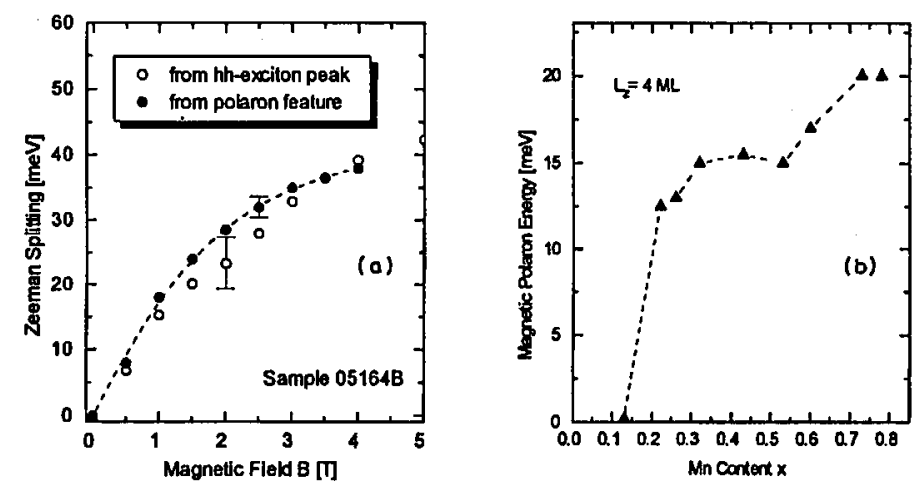

Fig. 2. (a) Total Zeeman splitting of heavy-hole excitons of a 6-ML-wide (20 $\AA$ ) $\mathrm{CdTe} / \mathrm{Cd}_{0.22} \mathrm{Mn}_{0.78} \mathrm{Te}$ single quantum well determined via the free exciton peak (open circles) and via the magnetic polaron feature (closed circles) in the photoluminescence excitation spectra. Dashed lines represent a Brillouin-fit. (b) Magnetic polaron energies in 4-ML-wide $\mathrm{CdTe} / \mathrm{Cd}_{1-x} \mathrm{Mn}_{x} \mathrm{Te}$ quantum wells as a function of Mn content; $T=1.6 \mathrm{~K}$. Data for QWs with $0.13 \leq x \leq 0.32$ are from Ref. [1].

Let us now summarize in Fig. $2 b$ the MP energies determined by selective excitation of localized excitons versus $\mathrm{Mn}$ content for $\mathrm{CdTe} / \mathrm{Cd}_{1-x} \mathrm{Mn}_{x} \mathrm{Te} \mathrm{QWs}$ with a well width of 4 MLs (for structures with slightly different well widths the MP energies are estimated by interpolation). We find an overall increase in the MP energy with increasing $x$. This result is surprising when one keeps in mind that 
in bulk $\mathrm{Cd}_{1-x} \mathrm{Mn}_{x} \mathrm{Te}$ with $x>0.13$ the magnetic susceptibility decreases with increasing $x$ [5] due to the antiferromagnetic pairing of neighboring Mn-spins. In addition, the penetration of the hh wave function into the semimagnetic barrier decreases with increasing $x$. Both of these factors should lead to a decrease in the MP energy with increasing $x$. However, the opposite dependence is found. We attribute this dependence to influences of the magnetic properties at the interface. The partial missing of the nearest-neighbors for $\mathrm{Mn}$ ions at the interface [2] and intermixing of the Mn concentration at the interfaces [3] are possible mechanisms. We expect for structures with $x>0.4$ that the last mechanism should dominate. The profile of the Mn content at the interfaces depends on the Mn content included in the barrier layers. For samples with high barriers an increased intermixing is expected due to an enhanced diffusion and slightly different growth conditions (substrate temperature and Te flux) for high Mn concentrations. By analyzing the Zeeman pattern a diffusion profile of the interface can be simulated [3]. For the 6-ML-wide QWs in the samples with $x=0.73$ and 0.78 it gives interface diffusion leı.gths of $4.2 \AA$ and $3.9 \AA$, respectively [6]. For the samples with lower Mn contents $(0.2 \leq x \leq 0.4)$ typical diffusion lengths of $2-3 \AA$ are found. From Fig. $2 \mathrm{~b}$ the correlation between diffusion length and MP energy is evident. Note that the sample with the largest diffusion length $(x=0.73)$ also exhibits the largest MP energies. Therefore, we attribute the observed high MP energies at the highest $x$ to a diffusion of Mn atoms into the CdTe well during growth.

In conclusion, we have studied magnetic polarons in good quality $\mathrm{CdTe} / \mathrm{Cd}_{1-x} \mathrm{Mn}_{x} \mathrm{Te}$ quantum wells for $0.4 \leq x \leq 0.8$. In these structures the magnetic polaron formation leads to the appearance of an additional line in the photoluminescence excitation spectra, which can be employed to determine the Zeeman splittings more exactly than by using the free exciton peak. Our experimental results evidence that the relatively large observed polaron energies sensitively reflect the $\mathrm{Mn}$ concentration profile at the interface.

We wish to thank Nguyen The Khoi, P. Kossacki, and J. Gaj for providing us with results of their interface studies prior to publication.

\section{References}

[1] D.R. Yakovlev, in: Festkörperprobleme/Advances in Solid State Physics, Ed. U. Rössler, Vol. 32, Vieweg, Braunschweig 1992, p. 251.

[2] W. Ossau, B. Kuhn-Heinrich, Physica B 184, 422 (1993).

[3] J.A. Gaj, W. Grieshaber, C. Bodin-Deshayes, J. Cibert, G. Feuillet, Y. Merle d'Aubigné, A. Wasiela, Phys. Rev. B 50, 5512 (1994).

[4] A.K. Zakrzewski, E. Janik, E. Dynowska, M. Leszczyński, M. Kutrowski, T. Wojtowicz, G. Karczewski, J. Bąk-Misiuk, J. Domagała, J. Kossut, Acta Phys. Pol. A 87, 433 (1995).

[5] S.B. Oseroff, R. Calvo, W. Giriat, Z. Fisk, Solid State Commun. 35, 539 (1980).

[6] Nguyen The Khoi, P. Kossacki, J. Gaj, private communication. 